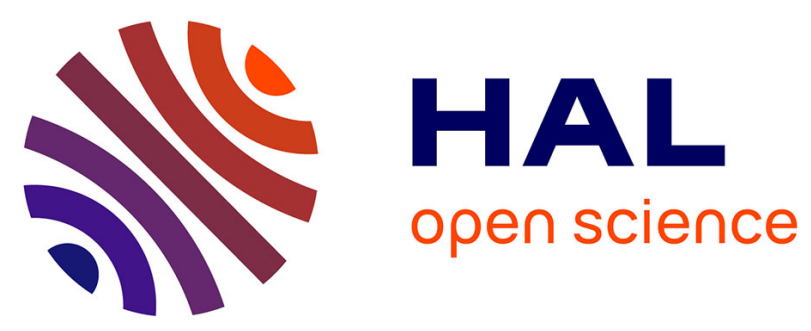

\title{
From linear amplification to triggered superradiance: illustrative examples of stimulated emission and polarization spectroscopy for sensitive detection of a pulsed excited forbidden transition
}

\author{
M. Bouchiat, J. Guéna, Ph. Jacquier, M. Lintz, L. Pottier
}

\section{To cite this version:}

M. Bouchiat, J. Guéna, Ph. Jacquier, M. Lintz, L. Pottier. From linear amplification to triggered superradiance: illustrative examples of stimulated emission and polarization spectroscopy for sensitive detection of a pulsed excited forbidden transition. Journal de Physique II, 1992, 2 (4), pp.727-747. 10.1051/jp2:1992162 . jpa-00247668

\section{HAL Id: jpa-00247668 https://hal.science/jpa-00247668}

Submitted on 1 Jan 1992

HAL is a multi-disciplinary open access archive for the deposit and dissemination of scientific research documents, whether they are published or not. The documents may come from teaching and research institutions in France or abroad, or from public or private research centers.
L'archive ouverte pluridisciplinaire HAL, est destinée au dépôt et à la diffusion de documents scientifiques de niveau recherche, publiés ou non, émanant des établissements d'enseignement et de recherche français ou étrangers, des laboratoires publics ou privés. 
Classification

Physics Abstracts

$32.70-32.80 \mathrm{~B}-42.65 \mathrm{G}-35.10 \mathrm{~W}$

\title{
From linear amplification to triggered superradiance: illustrative examples of stimulated emission and polarization spectroscopy for sensitive detection of a pulsed excited forbidden transition
}

\author{
M.A. Bouchiat, J. Guéna, Ph. Jacquier, M. Lintz and L. Pottier \\ Laboratoire de Spectroscopie Hertzienne $\left(^{\star}\right)$, Département de Physique de l'ENS, 24 rue \\ Lhomond, 75231 Paris Cedex 05, France
}

(Received 27 December 1991, accepted 17 January 1991)

\begin{abstract}
This paper presents our experimental strategy for detecting an electric-field controlled pulsed-excited highly forbidden transition such as the $6 \mathrm{~S}-7 \mathrm{~S}$ transition of the $\mathrm{Cs}$ atom. The amplification of a pulsed probe beam close to resonance for one hfs component of the $7 \mathrm{~S}-6 \mathrm{P}_{3 / 2}$ transition is monitored. Furthermore the polarization modification experienced by the probe beam as a result of its interaction with the vapor is meticulously analysed. In convincing examples we point out the high sensitivity and complete selectivity of the method for the angular anisotropy of the $7 \mathrm{~S}$ state, such as orientation or alignment created in the $6 \mathrm{~S}-$ $7 \mathrm{~S}$ excitation process. We illustrate the flexibility of the method by varying the pump-probe delay, or by changing the relative positions of the beams or the magnitude of the $E$-field that directly controls the optical gain. A smooth transition from linear amplification to triggered superradiance is observed which necessitates readjustment of the probe intensity for optimal detection. We demonstrate the vapor-induced enhancement of the amplification anisotropy, expected at high optical density.
\end{abstract}

Sensitive detection of excited atoms is a problem nowadays commonly solved by looking at their interaction with a radiation field. With the advent of tunable laser sources there are so many possibilities that the best choice is far from obvious. Transmission techniques using the pumping beam, or stimulated emission (or absorption) from the excited state using a second laser beam can now serve as substitutes for fluorescence detection. Which of these methods deserve the most consideration?

Looking backwards to the early days of Optical Pumping we learn an instructive lesson. In the seminal Kastler ideas [1] and in the pioneering experimental work that followed [2], the ground state orientation was detected through the polarization of the reemitted fluorescence light. However several years later, Dehmelt's proposal of directly observing the pumping beam after its transmission through the vapor [3] generated a renaissance in the field of optical

(*) Laboratoíre associé au CNRS (URA 18), à l'Ecole Normale Supérieure et à l'Université Pierre et Marie Curie. 
pumping. With this new technique it became possible to observe a high degree of atomic ground state orientation in conditions where the fluorescence light was depolarized or severely quenched by collisions in the excited state. A new kind of pumping mechanism, Dehmelt pumping, was born [4]. This example is a good illustration of the complementarity between the fluorescence and the transmission detection methods. Each one requires different optimization conditions in the preparation stage of the atoms to be detected, and produces a different kind of information. Therefore they cannot be substituted to one another indifferently. A restriction on the interest of the transmission technique is that a single beam is given two roles : pumping and detection. In some cases the roles may fall into conflict. This kind of difficulty was solved by making use of two beams : one intense pumping beam and one faint probe beam observed in transmission $[5,6]$. Then either beam could be optimized in intensity, polarization and frequency. The versatility of the experiments as well as the clarity of their interpretation were thus simultaneously upgraded [7].

Sensitive detection of excited atoms is one of the major difficulties encountered in today's problem of accurate measurement of parity violation in atoms [8]. As a definite example we take cesium. The detection problem concerns the $7 \mathrm{~S}$ atoms excited through the highly forbidden 6S-7S transition. In the first generation PV experiment fluorescence light emitted directly in the $7 \mathrm{~S}_{1 / 2}-6 \mathrm{P}_{1 / 2}$ fine structure transition was observed and its polarization analyzed. This method, reminiscent of the Kastler approach, although it has produced significant results [9], has the drawback of very low efficiency. Since our goal is to achieve a better experimental statistics, we have addressed the problem of active detection of the 7S state [10]. Namely during the lifetime of the excited state the atoms interact with another radiation field from a second tunable laser. In the present case this laser is tuned to one hfs component of the $7 \mathrm{~S}-6 \mathrm{P}_{3 / 2}$ transition. Then, i) the fluorescence which is induced by the pump laser in the $7 \mathrm{~S}-6 \mathrm{P}_{1 / 2}$ component is reduced whenever the second laser is resonant for one hfs component $[11,12]$ and $\mathrm{ii)}$ with sufficiently efficient $7 \mathrm{~S}$ pumping, the probe radiation may even be amplified [13].

Obviously the problem of making active optical detection efficient and sensitive is not restricted to the case of atomic PV measurements. This question has received very broad interest in the domains of molecular spectroscopy and chemical dynamics for the last ten years. The method we call inhibited fluorescence spectroscopy [11] has more than one common feature with Stimulated Emission Pumping (SEP), now a powerful vibration-rotation spectroscopy tool quite generally used for small polyatomics [14]. Independently, active detection was demonstrated quite early in the history of high resolution spectroscopy [15].

For several reasons we believe that the three-level lambda-type $6 \mathrm{~S}-7 \mathrm{~S}-6 \mathrm{P}$ system in cesium (Fig. 1) is a case of special interest, regardless of its connection with the PV problem :

i) The two $S$ states connected by the pump laser are of the same parity, the oscillator strength is only $4 \times 10^{-15}$, and the $6 \mathrm{~S}-7 \mathrm{~S}$ transition rate is easily governed by a static electric field E. The excitation probability is $P \propto \alpha^{2}|\mathbf{E} \cdot \epsilon|^{2}+\beta^{2}|\mathbf{E} \times \epsilon \cdot \sigma|^{2}$ where $\alpha$ and $\beta$ stand for the scalar and vector transition polarizabilities, $\epsilon$ for the pump polarization and $\sigma$ for the Pauli operator acting on the electronic spin state [16]. (The ratio $\alpha / \beta=-9.9$ is well determined [17]). Consequently, varying the field magnitude is a very convenient way of controlling the $7 \mathrm{~S}-6 \mathrm{P}$ population inversion. With intense pulsed excitation the transition rate can be made large enough so that the excited vapor becomes superradiant for the $7 \mathrm{~S}-6 \mathrm{P}$ transitions. We thus have a unique situation in which one can pass continuously from a regime of weak linear amplification to triggered superradiance, by just changing at will the oscillator strength without any modification in the damping rates. This leads to simply interpreted results.

ii) As it is conceived, our $6 \mathrm{~S}-7 \mathrm{~S}-6 \mathrm{P}$ experiment simultaneously demonstrates many of the possibilities of the active detection method. We have tried to take full advantage of all the 


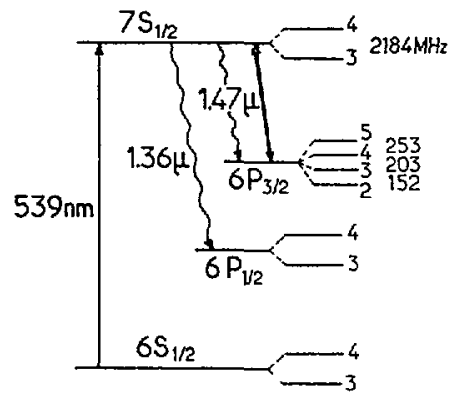

Fig. 1. - Scheme of the lower levels of cesium. Straight arrows : laser couplings. Wavy arrows : fluorescence.

parameters which specify both the pump and the probe beams. In particular we attach special importance to light polarization dependent effects. Practically all configurations of pump and probe polarizations have been realized. We will give several illustrations of the fruitful association of active detection with polarization spectroscopy $[6,19]$. In addition, when the optical medium becomes optically thick at the probe frequency, we have succeeded in demonstrating the additional attractive feature predicted before [10], an enhancement of the polarization dependent effects.

The method developed by the Zurich group also aiming at a sensitive detection of the $6 \mathrm{~S}-7 \mathrm{~S}$ transition [18] might, at first sight, look closely connected to our's : it consists in a two-laser experiment on the three level 6S-7S-15P system. However the Zurich experiment does not involve optical detection at all (the Cs $15 \mathrm{P}$ atoms are detected in a thermionic diode), it is performed with $\mathrm{cw}$ lasers and all polarization effects connected with an angular anisotropy of the 7S state are ignored. We prefer our approach because we believe in the importance of a direct observation of the $7 \mathrm{~S}$ atoms which have completed the $6 \mathrm{~S}-7 \mathrm{~S}$ one-photon transition.

It is the purpose of this paper to illustrate the possibilities opened up by the use of active detection with the example of the $6 \mathrm{~S}-7 \mathrm{~S}-6 \mathrm{P}$ system prepared with intense pulsed excitation. They can conveniently be classified according to the typical effects generated in the probe beam when the pump beam is turned on, and this motivates the division of this paper into the following six sections :

- Demonstrating active detection with resonant probe amplification

- Associating active detection and polarization spectroscopy

- Changing the time delay between pump and probe

- Changing the gain parameter : three amplification regimes

- Enhancing polarization dependent signals

- Varying the relative position of the two laser beams.

1. Demonstrating active detection with resonant probe amplification.

The experiment uses $10 \mathrm{~ns}$ long pulses to excite the $6 \mathrm{~S}-7 \mathrm{~S}$ transition $(\lambda \simeq 539 \mathrm{~nm})$. The frequency is stabilized and the spectral width is nearly Fourier limited so that tuning at the center of one hfs component can be achieved. A cw monomode tunable laser beam, collinear to the pump, probes the $7 \mathrm{~S}-6 \mathrm{P}_{3 / 2}$ transition at $1.47 \mu \mathrm{m}$. It is driven by a fast electrooptical 


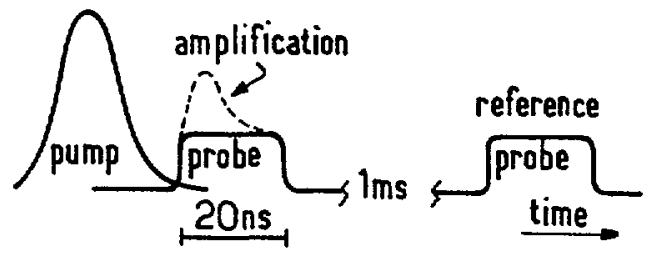

Fig. 2. - Typical experimental timing for the laser pulses.

switch $\left({ }^{1}\right)$ which gives high flexibility in the choice of operating conditions, namely pump-probe delay and probe duration. After the $7 \mathrm{~S}$ atoms have emitted their photon, they accumulate in the $6 \mathrm{P}_{3 / 2}$ state which is made long-lived by resonance radiation trapping. From previous observations [12] we know that the lifetime of the $6 \mathrm{P}$ population reaches several microseconds. Consequently it is essential to observe the probe only as long as stimulated emission dominates absorption. In typical working conditions the probe beam is switched on at the end of the pump pulse for an optimized duration $\sim 20 \mathrm{~ns}$ (see Fig.2). A second identical probe pulse, switched on $1 \mathrm{~ms}$ after the first one, monitors the transmission when no excited atoms remain and provides the reference $S$ needed to extract the vapor contribution. In this "bipulse" technique the vapor amplification factor is obtained at each pump pulse.

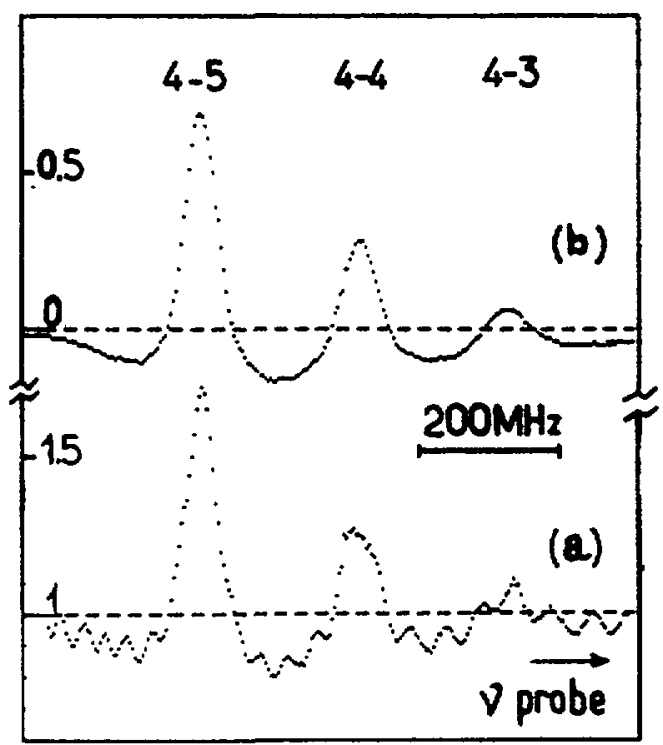

Fig. 3. - Amplification of the probe beam, vs. probe frequency : a) Transmitted probe signal $S_{\text {out }}$. The oscillations are due to etalon effects in an optical fiber. b) Amplification power $\left(S_{\text {out }}-S\right) / S$. This quantity is unaffected by probe intensity fluctuations or drifts. $n_{\mathrm{Cs}} \simeq 2.7 \times 10^{14} \mathrm{at} / \mathrm{cm}^{3}$, transverse $E$ field $280 \mathrm{~V} / \mathrm{cm}$. Exc. pulse $1 \mathrm{~mJ}$, resonant for $6 \mathrm{~S} F=4 \rightarrow 7 \mathrm{~S} F=4$. Probe nearly saturating.

$\left({ }^{1}\right)$ We use an integrated $\mathrm{LiNbO}_{3}$ directional coupler of $2 \mathrm{GHz}$ bandwidth, operated with typical voltages of only $10 \mathrm{~V}$ (generously provided by A. Carenco from CNET, Bagneux, France). 


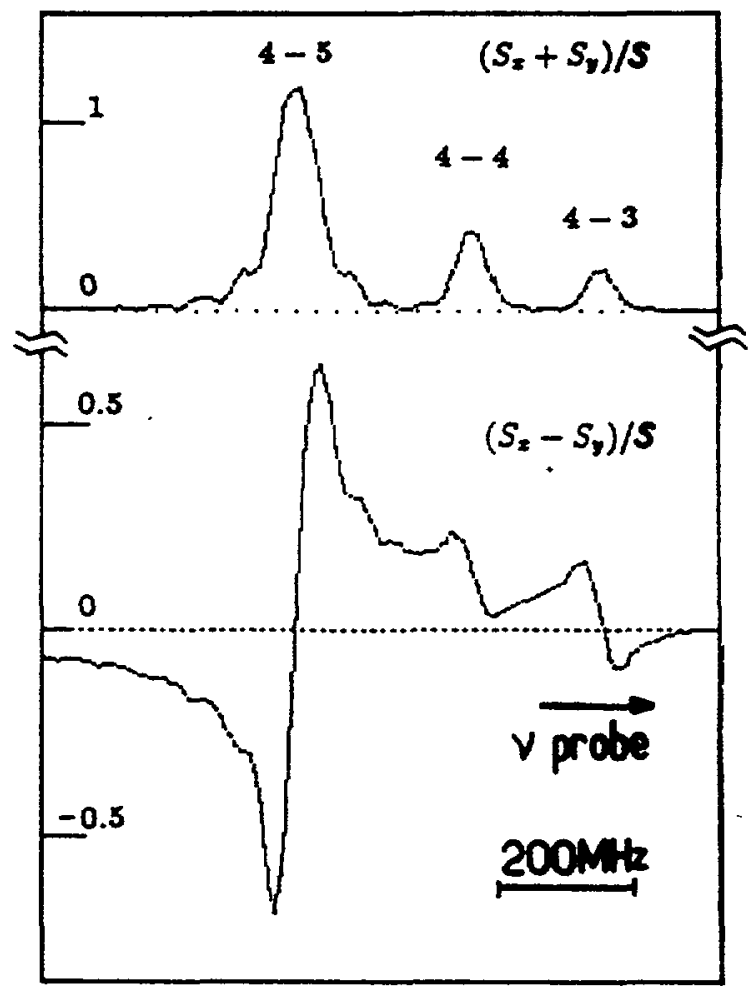

Fig. 4. - Top : Amplification power vs. probe frequency. Bottom : Optical rotation spectrum. $n_{\mathrm{Cs}} \simeq 2 \times 10^{14}$ at $/ \mathrm{cm}^{3}$; longitudinal $E$ field : $2.4 \mathrm{kV} / \mathrm{cm}$. Exc. pulse $400 \mu \mathrm{J}$, circularly polarized, resonant for $6 \mathrm{~S} F=3 \rightarrow 7 \mathrm{~S} F=4$.

Figure 3 illustrates the spectrum of the probe amplification when the probe frequency is swept over the hfs profile of the $7 \mathrm{~S} F=4 \rightarrow 6 \mathrm{P}_{3 / 2}$ transition. Comparison between figures $3 \mathrm{a}$ and $3 \mathrm{~b}$ clearly demonstrates the advantage of the "bipulse" technique for providing signals unaffected by probe intensity fluctuations. Several features of the recorded spectra are noteworthy :

i) Despite the very weak optical absorption at the pump wavelength $\left(\approx 10^{-7}\right)$, the amplification factor reaches $\approx 100 \%$ (Fig. 4 , top), and even more in the conditions where the vapor superradiates (see Sect.4).

ii) As expected from velocity selection by the collinear pump and probe beams the amplification displays sub-Doppler resolution : amplification occurs only if the pump and the probe are resonant for the same velocity class. As shown in figure 3 this makes it possible to completely resolve the $6 \mathrm{P}_{3 / 2}$ hyperfine structure. Note however that such a result requires to simultaneously satisfy two conflicting conditions : the spectral resolution of the lasers must of course be high, but so must also be their temporal resolution since we have to build a high 7S population in a time short compared to the $7 \mathrm{~S}$ state decay time. The pulse durations which satisfy both conditions actually belong to a narrow domain, having its center close to the actual conditions.

iii) The three lines in figure 3 are seen to appear on a negative Doppler-broadened profile of lower amplitude, whose relative height is observed to increase with the Cs density. This absorption due to thermalized $6 \mathrm{P}_{3 / 2}$ atoms appears unrelated to $6 \mathrm{~S}-7 \mathrm{~S}$ excitation, as shown by 
its persistence when the pump pulse is detuned from resonance, in which case the amplification peaks disappear. We have identified this effect as due to $6 \mathrm{P}$ excitation occuring during the pump pulse by indirect non-resonant processes (such as photoelectron production and electron bombardement of the ground state atoms). As shown below these processes do not affect the polarization dependent spectra.

\section{Associating active detection and polarization spectroscopy.}

In the case of the E-field controlled $6 \mathrm{~S}-7 \mathrm{~S}$ transition, the angular state of the excited atoms depends not only on the pump polarization but also on the E-field direction. The detection of angular properties of an atomic state by polarization analysis of a transmitted probe beam has a long history in optical-pumping experiments [4, 7]. The $6 \mathrm{~S}-7 \mathrm{~S}-6 \mathrm{P}$ system nicely illustrates here that the high sensitivity of this method is not restricted to $\mathrm{cw}$ laser operation. "Our experimental procedure, specifically conceived for the case of pulsed operation with inherently large source instabilities, is shown to achieve sensitive measurements of the outgoing probe polarization. Furthermore, the $6 \mathrm{~S}-7 \mathrm{~S}-6 \mathrm{P}$ system will show the following crucial feature : the polarization effects, completely insensitive to the 6P atoms, are characteristic of the 7S angular properties. This detection appears not only selective but also flexible since it allows the choice of measuring any 7S orientation or alignement. In addition, because amplification factors as large as $100 \%$ can be reached, there is no dilution in the transfer of the $7 \mathrm{~S}$ angular anisotropy to the modification of the probe polarization.

The polarization $\epsilon_{\mathrm{pr}}$ of the probe at the output of the cell is analyzed by a polarizing beamsplitter cube with two detection channels corresponding to the two orthogonally polarized output beams [20]. With an incoming probe beam chosen linearly polarized the axes of the cube are oriented so as to analyze the two polarizations at $\pm 45^{\circ}$ from $\epsilon_{\mathrm{pr}}$. The amplitude of each output signal is proportional to the number of photons detected during the pulse. With the excitation laser off (second probe pulse in the "bipulse" technique, see Fig. 2), the two outputs balance each other exactly and their sum $S$ serves as a reference. With the excitation laser on (first probe pulse), subtraction of the reference from each output provides the two amplification signals $S_{x}$ and $S_{y}$. The normalized sum $\left(S_{x}+S_{y}\right) / S$ measures the amplification factor of the vapor. Simultaneously the normalized differential signal $\left(S_{x}-S_{y}\right) / S$ gives the polarization rotation angle correlated with the pulsed excitation. In balanced operation, taking the difference of the two channels leads to high rejection of the technical common noise and brings down the noise close to the shot noise limit [20].

As a first illustration of the polarization dependent effects, figure 4 concerns the situation where the pump beam is circularly polarized, thus giving rise to a $7 \mathrm{~S}$ orientation directed along the beams. The pump frequency is kept resonant for one hfs component $\left(6 \mathrm{~S}_{1 / 2} F=3 \rightarrow\right.$ $\left.7 \mathrm{~S}_{1 / 2} \quad F=4\right)$. The amplification factor and the polarization dependent signal are plotted versus the probe frequency swept across the $6 \mathrm{P}_{3 / 2} \mathrm{hfs}$. The polarization signal exhibits three dispersive line-shaped resonances whose relative signs and heights agree with the calculated optical rotation spectrum resulting from the orientation of the 7S state. (In [12] similar agreement was found for the $\mathrm{cw}$ spectra). The small wiggles noticeable on the wings of the lines simply result from the (time) Fourier transform of the rectangular shaped detection function.

The spectra in figure 5 were recorded in somewhat extreme conditions (high Cs density) where the Doppler free amplification lines are almost dominated by the absorption due to the thermalized $6 \mathrm{P}_{3 / 2}$ atoms. In such conditions the amplification spectrum looks messy and is difficult to interpret. In marked contrast the differential signal $\left(S_{x}-S_{y}\right)$ is clean. It exhibits no Doppler broadened contribution, an indication that $6 \mathrm{P}$ atoms do not contribute. The same 
conclusion is independently deduced from records taken either using a non-resonant pump beam or with a probe laser tuned for the unpopulated hyperfine level of $7 \mathrm{~S}$. The polarization dependent signal indeed achieves complete selectivity of $7 \mathrm{~S}$ atoms alone.

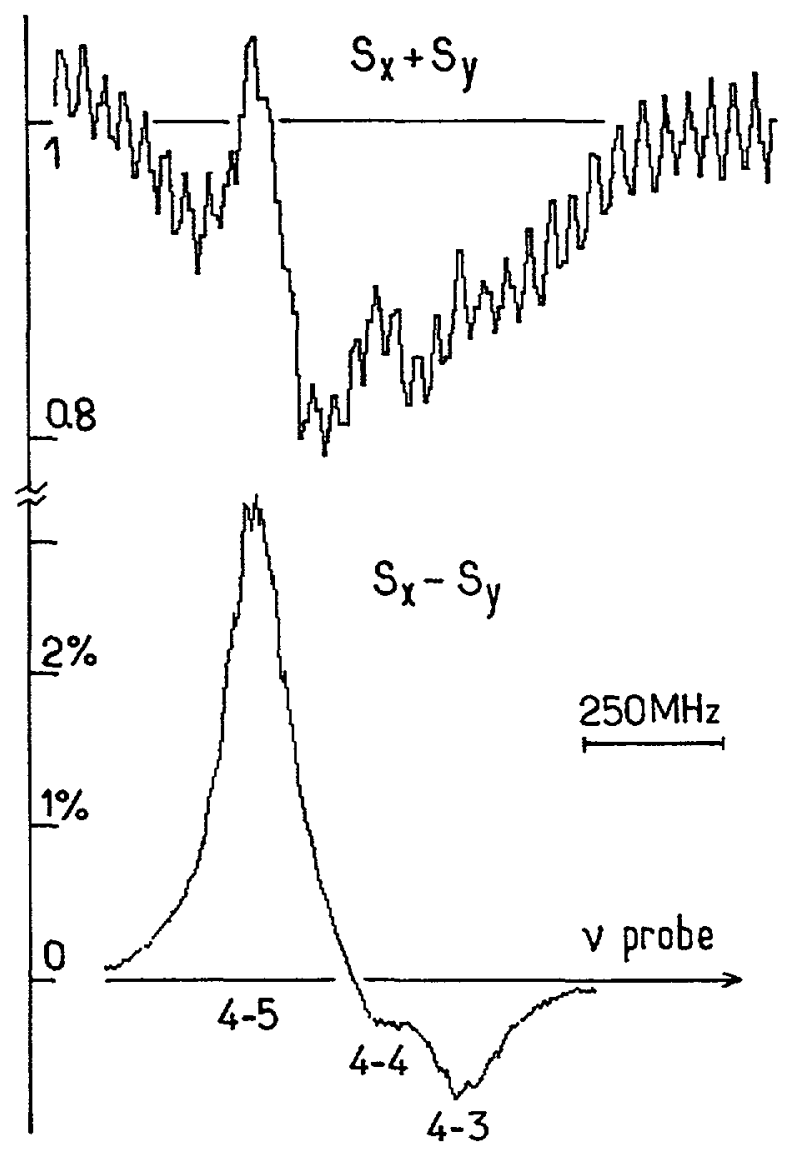

Fig. 5. - Selectivity of the difference signal (Bottom) even when the sum signal (Top) is overwhelmed in a background. Top : unnormalized transmitted intensity (no reference subtraction). Bottom : difference signal recorded with circular analysis ( $\lambda / 4$ plate inserted before the polarimeter), in units of probe intensity. $n_{\mathrm{Cs}} \simeq 9.5 \times 10^{14}$ at $/ \mathrm{cm}^{3}$, transverse $E$ field $: 65 \mathrm{~V} / \mathrm{cm}$. Exc. pulse circularly polarized, resonant for $F=4 \rightarrow F=4$.

Figure 5 was taken with a circularly polarized pump beam as previously (in Fig.4) but with a circularly polarized analysis of the probe beam (insertion of a $\lambda / 4$ plate just before the analyzing cube). On the other hand, the technique of the probe bipulse was not yet in place. The advantages gained with the bidifferential method were appreciated later on. Not only does this technique eliminate fluctuations of the amplification signal but it also proves invaluable for precise polarization measurements. Slight imbalances of the polarimeter independent of the atomic system, in particular the polarization defects of all the optics which may evolve in time, are detected at each pulse and can be corrected for, leaving the anisotropy of the vapor 
well distinguished. These possibilities are fully exploited in the experimental results presented next.

Figure 6a illustrates a situation in which the probe frequency is kept resonant for the hfs $7 \mathrm{~S}_{1 / 2} F=4 \rightarrow 6 \mathrm{P}_{3 / 2} F^{\prime}=4$ component, while sweeping the pump frequency $\nu_{\mathrm{ex}}$ through the Doppler $6 \mathrm{~S} F=3 \rightarrow 7 \mathrm{~S} F=4$ absorption profile. Atoms with non-zero longitudinal velocities are thus brought to the $7 \mathrm{~S}$ state in proportion to the ground state maxwellian distribution. Since the probe is tuned at resonance for the zero velocity class the amplification factor exhibits a narrow (Doppler-free) maximum at $\nu_{\mathrm{ex}}$ resonant for the same velocity class. In addition two bumps appear on each side of the main resonance. They correspond to the $7 \mathrm{~S}$ atoms whose non-zero velocity makes them resonant for either of the two adjacent hfs $4 \rightarrow 3$ or $4 \rightarrow 5$ components of $7 \mathrm{~S}_{1 / 2} \rightarrow 6 \mathrm{P}_{3 / 2}$, distant from the $4 \rightarrow 4$ line by only 203 and $-253 \mathrm{MHz}$ respectively.
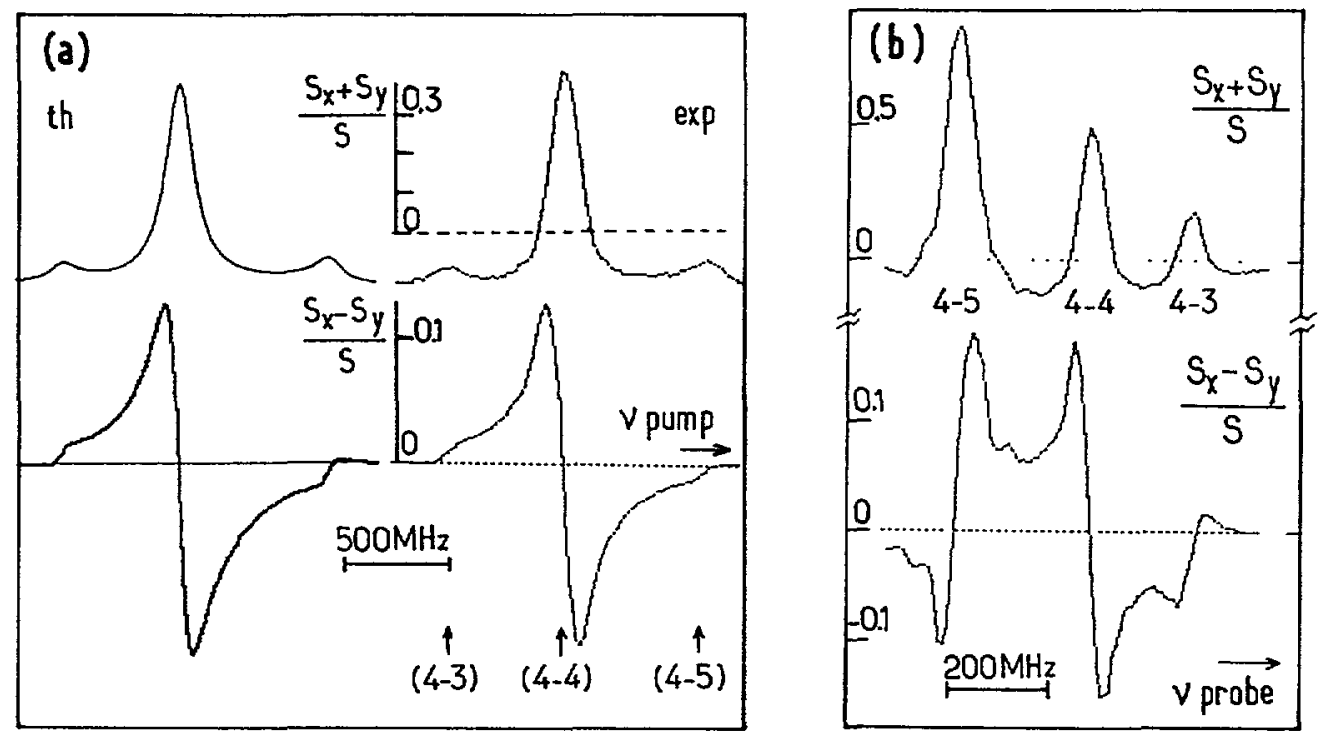

Fig. 6. - Spectra of the linear birefringence signal: a) Amplification power (top) and birefringence signal (bottom) vs. excitation frequency swept across the $F=3 \rightarrow F=4$ component. The resonances indicated are those of the probe with the Doppler-shifted excited velocity classes. $n_{\mathrm{Cs}} \simeq 10^{14} \mathrm{at} / \mathrm{cm}^{3}$, longitudinal $E$ field : $2 \mathrm{kV} / \mathrm{cm}$. Exc. pulse : $1 \mathrm{~mJ}$, linear polar. Probe kept resonant for 4-4; circular polar. b) Same signals as in a) vs. probe frequency. Same conditions, except probe circular polarization of opposite sign. Pump frequency kept resonnant for $6 \mathrm{~S}, F=3 \rightarrow 7 \mathrm{~S}, F=4$ excitation.

In the conditions of figure 6 the pump polarization $\epsilon_{\mathrm{ex}}$ is linear and the $\mathbf{E}$ field is parallel to the direction $\widehat{\mathbf{k}}$ of both beams. Consequently the excitation process possesses a symmetry with respect to the plane $\left(E, \epsilon_{\mathrm{ex}}\right)$. Therefore we expect the principal axes of the vapor anisotropy to be $\widehat{\boldsymbol{\epsilon}}_{\text {ex }}$ and the orthogonal direction $\widehat{\mathbf{k}} \times \widehat{\boldsymbol{\epsilon}}_{\text {ex }}$. In particular the probe beam should experience a linear birefringence of the same axes, whenever it approaches resonance. Since this effect concerns the real part of the refraction index it is expected to have a dispersive line shape. This is well demonstrated in the lower part of figure 6 . The polarimeter axes have been oriented at $45^{\circ}$ from the anisotropy axes. The polarization of the incoming probe beam, chosen circular, 
becomes elliptic under the effect of the vapor linear birefringence. This causes an imbalance at the output of the polarimeter. As shown in figure $6 a$ the observed effect agrees well, in size and sign, with theoretical predictions.

For comparison, figure $6 \mathrm{~b}$ shows the corresponding experimental spectra when the probe (instead of the pump) frequency is swept across the 7S $F=4 \rightarrow 6 \mathrm{P}_{3 / 2}$ hfs, the pump frequency now being kept resonant for the zero-velocity class on $6 \mathrm{~S} F=3 \rightarrow 7 \mathrm{~S} F=4$. The three resonances, now associated with the same class of $7 \mathrm{~S}$ atoms, have comparable sizes, and angular factors alone explain the remaining differences. (These factors can be calculated using the formulas given in [12], appendix B).

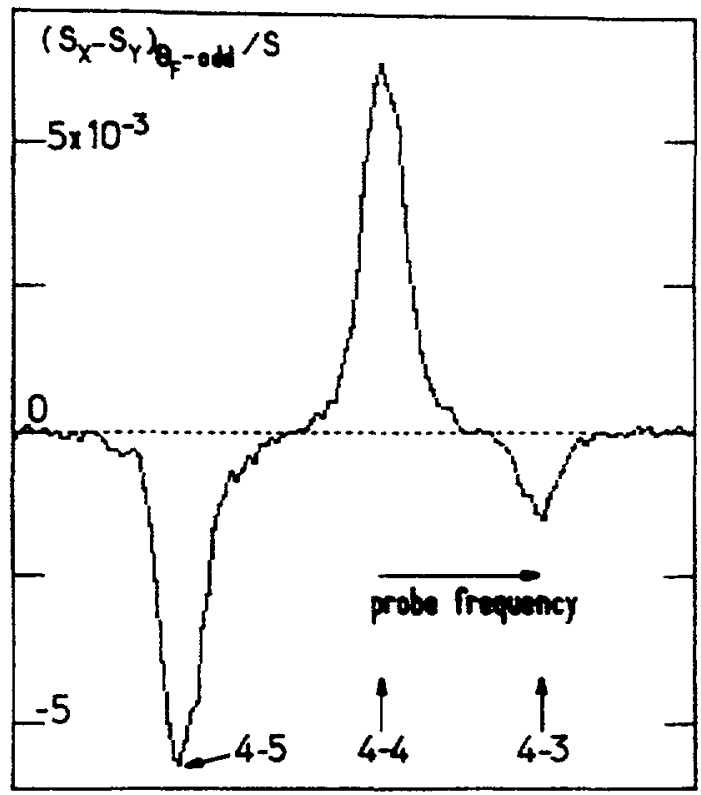

Fig. 7. - Detection of a small linear dichroism. The angle between pump and probe linear polarizations is modulated by a small angle $\pm \theta_{\mathrm{F}}$. The $\theta_{\mathrm{F}}$-odd difference signal is plotted vs. probe frequency. Each point is averaged over 18 shots. $\theta_{\mathrm{F}}=11 \times 10^{-3} \mathrm{rad}$. Longit. $E$-field : $2.5 \mathrm{kV} / \mathrm{cm}$. Probe amplification factor $\simeq 1$. Exc. pulse : $1.1 \mathrm{~mJ} . n_{\mathrm{Cs}} \simeq 10^{14} \mathrm{at} / \mathrm{cm}^{3}$.

The high sensitivity of the method is illustrated in figure 7, where an anisotropy of a few $10^{-3}$ is shown to be detected well above the noise despite a short integration time. In this experiment, a longitudinal $\mathbf{E}$ field is applied and the direction of the pump linear polarization $\epsilon_{\mathrm{ex}}$ is tilted with respect to the direction of the probe linear polarization $\epsilon_{\mathrm{pr}}$ by a small angle $\theta_{\mathrm{F}}$ (induced by a Faraday glass modulator). Consequently the principal axes of the 7S anisotropy, and therefore the axes of the linear dichroism at the probe frequency, are also tilted, by the same angle $\theta_{\mathrm{F}}$. This results in a $\theta_{\mathrm{F}}$-odd contribution to the differential signal of the polarimeter (whose axes are at $\pm 45^{\circ}$ from $\widehat{\epsilon}_{\mathrm{pr}}$ ). The spectrum of the $\theta_{\mathrm{F}}$-odd signal vs. probe frequency, shown in figure 7 , agrees with the prediction. At resonance the sensitivity corresponds to a signal/noise ratio of unity in $1 \mathrm{~s}$ (or 12 shots) for $\theta_{\mathrm{F}} \simeq 10^{-4}$

Concerning the polarization spectra shown above, we draw attention to the large difference in the relative weights and signs of the three $6 \mathrm{P}_{3 / 2} \mathrm{hfs}$ components according to whether the 
detected dichroism (or birefringence) is circular or linear : look for instance at the difference between figure 4 (optical rotation) and figure $6 \mathrm{~b}$ (linear birefringence) or between figures 5 and 7 (circular and linear dichroism). This is well understood on theoretical grounds [12], assuming that these anisotropies result from the atomic orientation or alignment created by the pump beam, in the absence of any magnetic field (a condition fulfilled here). The spectral shape of the polarization dependent signal is therefore a reliable means to confirm the type of vapor anisotropy experienced by the probe beam.

In this section, we have illustrated how the balanced polarimeter enables one to measure different vapor anisotropies depending on its orientation and on the probe polarization. A formal treatment shows that, in fact, all kinds of anisotropy can be measured. A classification of small anisotropies can be made systematic and complete in the formalism of the Stokes parameters often used to describe light polarization [21]. The steady state propagation through the excited vapor can be described by a complex $2 \times 2$ transfer matrix $M$ which relates the polarizations at the input and at the output of the cell $\epsilon_{\mathrm{pr}}^{\text {out }}=M \epsilon_{\mathrm{pr}}^{\mathrm{in}}$. We suppose hereafter that $\left|\epsilon_{\mathrm{pr}}^{\text {out }}-\epsilon_{\mathrm{pr}}^{\mathrm{in}}\right|$ is small and can be treated as a first order perturbation. Choosing two real, unitary, orthogonal vectors $(\widehat{\mathbf{x}}, \widehat{\mathbf{y}})$ perpendicular to the probe wavevector $\widehat{\mathbf{k}}_{\mathrm{pr}}$ as a basis for the polarizations, we use the Pauli and unity matrices as a complete basis to develop the matrix $M$ :

$$
M=\mathbb{1}+\left(\gamma_{0}+i \alpha_{0}\right) \mathbb{1}+\left(\gamma_{1}+i \alpha_{1}\right)\left(\begin{array}{ll}
0 & 1 \\
1 & 0
\end{array}\right)+\left(\gamma_{2}+i \alpha_{2}\right)\left(\begin{array}{cc}
0 & -i \\
i & 0
\end{array}\right)+\left(\gamma_{3}+i \alpha_{3}\right)\left(\begin{array}{cc}
1 & 0 \\
0 & -1
\end{array}\right)
$$

where all coefficients $\gamma_{i}, \alpha_{i}$ are real. Apart from $\alpha_{0}$ and $\gamma_{0}$, related to the isotropic part of the index and gain of the vapor, we have introduced $\left(\widehat{\mathbf{u}} \equiv \frac{\widehat{\mathbf{x}}+\widehat{\mathbf{y}}}{\sqrt{2}}, \widehat{\mathbf{v}} \equiv \frac{\widehat{\mathbf{x}}-\widehat{\mathbf{y}}}{\sqrt{2}}\right)$

$\alpha_{1}$, the birefringence with axes $(\hat{\mathbf{u}}, \widehat{\mathbf{v}})$,

$\alpha_{2}$, the optical rotation,

$\alpha_{3}$, the birefringence with axes $(\widehat{\mathbf{x}}, \widehat{\mathbf{y}})$,

$\gamma_{1}$, the plane dichroism with axes $(\widehat{\mathbf{u}}, \widehat{\mathbf{v}})$

$\gamma_{2}$, the circular dichroism,

$\gamma_{3}$, the plane dichroism with axes $(\widehat{\mathbf{x}}, \widehat{\mathbf{y}})$.

$\alpha_{1}$ and $\gamma_{1}$ arise from an excited state alignment of axes $(\widehat{\mathbf{u}}, \widehat{\mathbf{v}}), \alpha_{3}$ and $\gamma_{3}$ from an alignment of axes $(\widehat{\mathbf{x}}, \widehat{\mathbf{y}}), \alpha_{2}$ and $\gamma_{2}$ from an orientation along $\widehat{\mathbf{k}}_{\mathrm{pr}}\left({ }^{2}\right)$. Our polarimeter in balanced mode operation makes it possible to disentangle the different quantities $\alpha_{i}$ and $\gamma_{i}$. Measurements have to be performed in three configurations with polarimeter eigenvectors constituting a complete basis in the 3-dimensional Stokes parameter space : i) $\widehat{\mathbf{x}}, \widehat{\mathbf{y}}$; ii) $\widehat{\mathbf{u}}=\frac{\widehat{\mathbf{x}}+\widehat{\mathbf{y}}}{\sqrt{2}}, \widehat{\mathbf{v}}=\frac{\widehat{\mathbf{x}}-\widehat{\mathbf{y}}}{\sqrt{2}}$; iii) $\hat{f}=\frac{\widehat{x}+i \widehat{y}}{\sqrt{2}}, \hat{=}=\frac{\widehat{x}-i \widehat{y}}{\sqrt{2}}$ In each configuration the probe polarization is oriented along either of the two directions that ensure exact balance without pump beam.

Table I summarizes the expressions of the differential signal at the polarimeter output in each case. Indeed, all of the coefficients can be extracted, even with a certain redundancy if a complete set of measurements can be realized.

$\left({ }^{2}\right)$ Using a magnetic field to rotate the angular state via the Hanle effect gives access to the other components of the orientation or alignment. 
3. Cl..unging the time delay between pump and probe.

In this section we illustrate the possibilities offered by varying the pump-probe delay in three specific examples.

Table I. - Expressions for the signal $\left(S_{x}-S_{y}\right) / 2 S$ at the output of the polarimeter in balanced operation, for a complete set of orientations. $\left(\hat{\mathbf{e}}_{1}, \hat{\mathbf{e}}_{2}\right)$ eigenaxes of the polarimeter; $\hat{\mathbf{e}}_{\mathrm{pr}}^{\text {in }}$ polarization of incoming probe; $(\hat{\mathbf{u}}, \hat{\mathbf{v}})$ and $(\hat{+}, \hat{-})$ defined in text.

\begin{tabular}{|c|c|c|c|}
\hline $\begin{array}{l}\left(\hat{\mathrm{e}}_{1}, \hat{\mathrm{e}}_{2}\right) \\
\hat{\boldsymbol{\epsilon}}_{\mathrm{pr}}^{\mathrm{in}}\end{array}$ & $(\hat{\mathbf{x}}, \hat{\mathbf{y}})$ & $(\hat{\mathbf{u}}, \hat{\mathbf{v}})$ & $(\hat{t}, \hat{-})$ \\
\hline $\begin{array}{l}\hat{\mathbf{x}} \\
\hat{\mathbf{y}} \\
\hat{\mathbf{u}} \\
\hat{\mathbf{v}} \\
\hat{+} \\
\hat{-}\end{array}$ & $\begin{array}{l}\gamma_{3}+\alpha_{2} \\
\gamma_{3}-\alpha_{2} \\
\gamma_{3}-\alpha_{1} \\
\gamma_{3}+\alpha_{1}\end{array}$ & $\begin{array}{l}\gamma_{1}-\alpha_{2} \\
\gamma_{1}+\alpha_{2} \\
\gamma_{1}+\alpha_{3} \\
\gamma_{1}-\alpha_{3}\end{array}$ & $\begin{array}{l}\gamma_{2}+\alpha_{1} \\
\gamma_{2}-\alpha_{1} \\
\gamma_{2}-\alpha_{3} \\
\gamma_{2}+\alpha_{3}\end{array}$ \\
\hline
\end{tabular}

3.1 OPTIMIZING THE VAPOR AMPLIFICATION POWER. - As expected intuitively, for collecting a large atomic signal it is essential that the probe not be established too late. Figure 8 shows that, in typical experimental conditions, maximum probe amplification occurs when the probe pulse starts close to the peak of the pump pulse. It then decreases rapidly at increasing delays. This behaviour cannot simply be explained by a loss of $7 \mathrm{~S}$ excited atoms due to spontaneous emission which would involve a longer time scale (the natural $7 \mathrm{~S}$ lifetime is $48.5 \mathrm{~ns}$ ). Neither can it be explained by the spurious absorption due to a $6 \mathrm{P}$ population. Indeed in figure 8 the absorption, as measured with non resonant pump pulses, increases smoothly with the delay and remains small. We believe that a possible explanation may involve in-phasing of individual atomic dipoles by the probe field, all the more efficient when spontaneous emission had not time enough to occur, one characteristic feature of the triggered superradiance discussed in the next section. We plan to investigate this question with a more judicious utilization of the optical switch gating the probe beam. One can attempt to search for a different interpretation in terms of a coherent two-photon process. We have previously discussed this process [12], though in the context of $\mathrm{cw}$ laser excitation, and found it negligible.

3.2 Demonstrating SMALL LIGHT-SHIFTS. - The intense radiation field of the pump laser needed to create population inversion in a short time is responsible for ac Stark shifts. At first sight such effects seem to be of minor importance here since we are interested neither in frequency measurements nor in high resolution spectroscopy. Nevertheless light-shifts might start to affect the polarization measurements if they were source of signal loss or signal noise. For instance, whenever the pump and probe pulses overlap, the probe radiation field experiences an atomic system whose level positions adiabatically shift during observation. To evaluate the practical importance of the effect, its magnitude can be calculated, but with some complications coming from geometrical and time averaging. This is why we found it valuable to quickly obtain an experimental answer. 


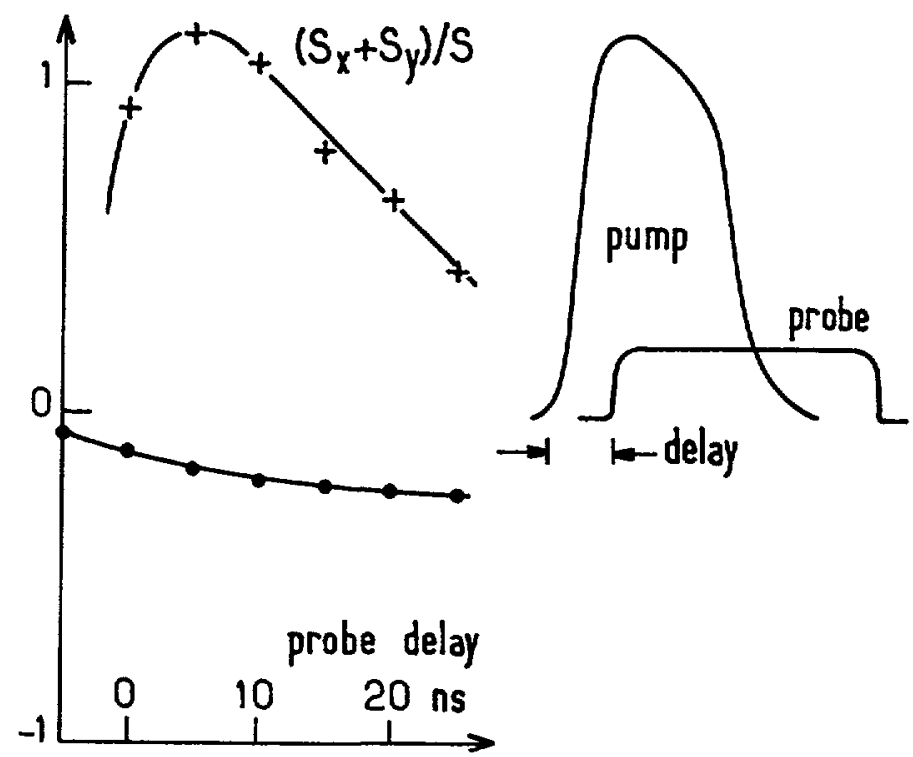

Fig. 8. - Amplification power vs. pump-probe delay with pump laser resonant for $6 \mathrm{~S}, F=3 \rightarrow 7 \mathrm{~S}$, $F=4(+)$ or non resonant $(\bullet)$. In both cases, the probe is kept resonant for $7 \mathrm{~S}, F=4 \rightarrow 6 \mathrm{P}_{3 / 2}, F=5$. Longit. $E$-field $2 \mathrm{kV} / \mathrm{cm}$. Exc. pulse $1 \mathrm{~mJ}$. $n_{\mathrm{Cs}} \simeq 2 \times 10^{14} \mathrm{at} / \mathrm{cm}^{3}$. Right : definition of the delay, at scale.

As shown in the preceding section (Figs. 4 and 6), optical rotation and birefringence created by the pump beam provide large dispersive shaped signals. As is well known, vanishing at the line center accurately determines the transition frequency, here averaged over the sample of atoms detected. The probe frequency that corresponds to a zero of this signal is found not to be the same when the probe is switched on during or after excitation. The measured difference is the light-shift of the $7 \mathrm{~S}-6 \mathrm{P}_{3 / 2}$ transition caused by the pump beam (averaged over space and time). The observed shift for a pump energy density of $\$ 1 \mathrm{~mJ} / \mathrm{mm}^{2}$ is always $<5 \mathrm{MHz}$, which is small enough not to be the source of any special concern presently.

3.3 DETECTING A SECONDARY RADIATIVE PROCESS INDUCED BY THE PUMP BEAM. - Intrigued by the origin of the non-resonant process responsible for a $6 \mathrm{P}$ state population induced by the pump pulse, we have probed the $6 \mathrm{P}$ population time evolution after a pump pulse. This was done without the electric field so as not to populate the 7S state. Figure 9 shows the measured absorption of the ( $20 \mathrm{~ns}$ long) probe pulse as it is delayed in time with respect to the pump pulse : the $6 \mathrm{P}$ population builds-up at a microsecond time scale and then decays on a longer scale. Also shown in figure 9 is the fit to the experimental data by the difference of two exponentials with time constants $1.25 \mu$ s and $3.2 \mu$ s respectively. The time constant for decay agrees well with the value $\sim 3 \mu$ s expected from our previous measurements of the damping time of the $6 \mathrm{P}_{3 / 2}$ population in similar conditions of resonance radiation trapping (high atomic density) [12]. On the other hand the rising time.constant $(1.25 \pm 0.05) \mu$ s matches well the $5 \mathrm{D}_{5 / 2}$ natural lifetime [22]. Indeed, according to the work of Collins et al. [23] the excitation of the Cs 5D state is expected to originate from the photolysis of cesium dimers in the laser frequency range 530-600 nm covering the 6S-7S resonance frequency (Fig. 10). 


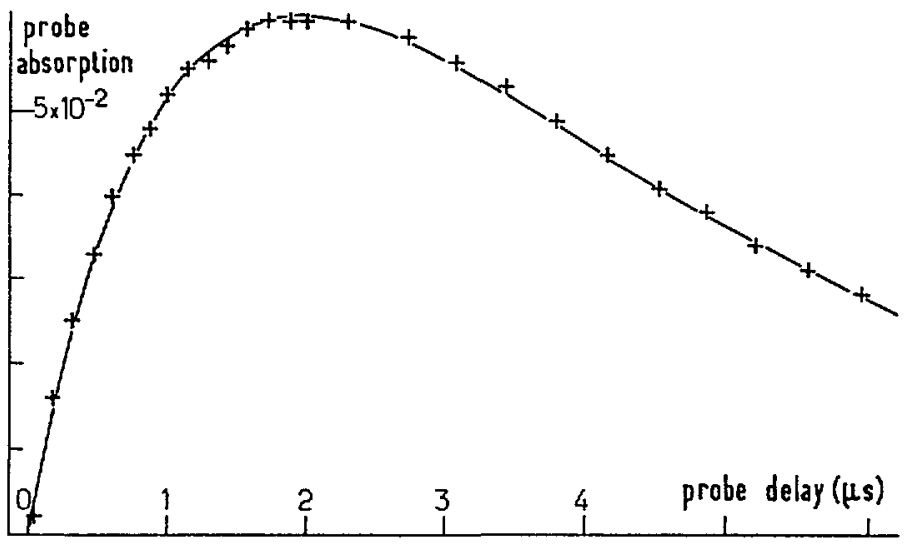

Fig. 9. - Absorption of the 20 ns long probe pulse following a pump pulse in absence of $E$-field, vs. pump-probe delay. Lasers resonant for $6 \mathrm{~S}, F=3 \rightarrow 7 \mathrm{~S}, F=4 \rightarrow 6 \mathrm{P}_{3 / 2}, F=5$. Pump energy $1.2 \mathrm{~mJ} . n_{\mathrm{Cs}} \simeq 10^{14} \mathrm{at} / \mathrm{cm}^{3} .(+)$ Experimental points. The curve is a fit by the difference of two exponentials with rising and decreasing time constants $1.3 \mu$ s and $3.2 \mu$ s respectively.

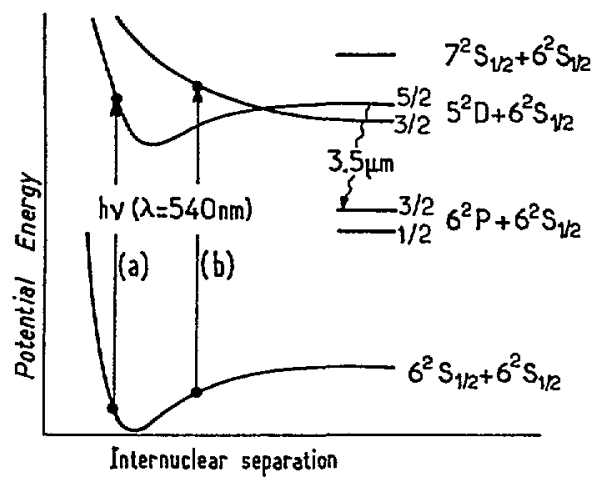

Fig. 10. - Schematic representation of the photolysis of $\mathrm{Cs}_{2}$ by photons of wavelength $\sim 540 \mathrm{~nm}$. The process occurs either by predissociation (channel a) or by direct dissociation (channel b).

Further consequences of this photolysis process in our operating conditions are currently being investigated and will be published elsewhere.

\section{Three amplification regimes.}

Of all the parameters (pump energy, cesium density...) affecting the 7S population, the electric field turns out to be a particularly convenient tool for its control. Its effect is simply quadratic, $N_{7 \mathrm{~S}} \sim E^{2}$, and allows us to explore three different amplification regimes : linear amplification for low densities of excited atoms, triggered superradiance for high densities, and then spontaneous superradiance when the probe beam is masked. 
These are obviously not three completely exclusive regimes. One can pass continuously from a situation in which atoms contribute individually to the amplification of the probe beam to a situation in which a macroscopic dipole, sum of all the individual atomic dipoles, forms and accelerates the de-excitation. The latter case corresponds to superradiance [24-26].

4.1 BRIEF STUDY OF THE SPONTANEOUS SUPERRADIANCE. - The intrinsically random nature of spontaneous superradiance deprives it of the stability necessary for sufficient sensitivity in the detection. This is why it is essential to know how to prevent it, and it is to this end that we have masked the probe and briefly studied this phenomenon.

Above a first threshold, a single superradiant pulse is observed, at $1.47 \mu \mathrm{m}\left(7 \mathrm{~S} \rightarrow 6 \mathrm{P}_{3 / 2}\right.$ transition). Above a second threshold, a second less intense pulse is emitted a few nanoseconds later on the $7 \mathrm{~S} \rightarrow 6 \mathrm{P}_{1 / 2}$ transition at $1.36 \mu \mathrm{m}$ (selected by a filter). The pulses at $1.47 \mu \mathrm{m}$ and $1.36 \mu \mathrm{m}$ are both observed at each shot.

Figure 11 shows that the threshold at $1.47 \mu \mathrm{m}$ is rather low, corresponding to a rather small number of excited atoms : $\sim 6 \times 10^{8}$ in an emitting volume $30 \mathrm{~mm}$ long and $1 \mathrm{~mm}$ in diameter. The reason is the near-absence of Doppler dephasing between the dipoles (due to the narrow spectral width of the excitation source). Indeed in this case one can assume that the threshold corresponds to the condition in which the superradiant pulse must occur before the atoms have spontaneously decayed from $7 \mathrm{~S}$. Using the well known formulas for the superradiance delay [26] :

$$
\begin{aligned}
& t_{\mathrm{D}}=T_{\mathrm{SR}} \log N \\
& T_{\mathrm{SR}}=\frac{8 \pi}{3} \frac{a^{2}}{N \lambda^{2} \Gamma}
\end{aligned}
$$

(here $\Gamma$ must be taken as the partial width of the most probable of all 7S-6P hyperfine transitions, $a$ is the diameter of the pumping beam, $\lambda=1.47 \mu \mathrm{m}$ the wavelength and $N$ the number of excited atoms), one finds a threshold : $N_{Z} 2 \times 10^{8}$. Its compatibility with the experimental value confirms that there are few spurious dephasings $\left({ }^{3}\right)$. It is noteworthy that the superradiant spontaneous emission gives rise to a lower detection efficiency than one would have with either amplification or triggered superradiance and that one no longer has a choice of the hyperfine transition for detection.

Figure 12 indicates the variation of the time delay of the spontaneous superradiance with the square of the electric field (that is, the number of excited atoms). As expected, it decreases when this number increases, but the delay of the emission is always longer for emission to $6 \mathrm{P}_{1 / 2}$ than for that to $6 \mathrm{P}_{3 / 2}$. The limiting value ( $\left.\sim 15 \mathrm{~ns}\right)$ seen in the figure is due to the response time of the photodiode. Clearly some inhibition process of the emission towards $6 \mathrm{P}_{3 / 2}$ prevents complete depopulation of the $7 \mathrm{~S}_{1 / 2} F=4$ state via the most probable channel, and permits subsequent emission towards $6 \mathrm{P}_{1 / 2}$. Such an incomplete desexcitation of an ensemble of atoms may be due to destructive interatomic interference called subradiance, which has been observed in a demonstrative experiment performed at Laboratoire Aimé Cotton [27].

Using a faster photodiode it has been possible to observe situations in which the medium emits superradiance at $\lambda=1.47 \mu \mathrm{m}$ even before the end of the excitation pulse. A second, less intense pulse at the same wavelength may follow the first, due to the re-buildup of the $7 \mathrm{~S}$ population (similar phenomena have been described in Ref. [25]).

$\left({ }^{3}\right)$ Introducing the field gain per unit time and unit length, calculated in reference [10], we note that $g L T_{\mathrm{SR}}=0.3$. Consequently the intensity amplification of a resonant beam over the total length is close to unity for a time interval equal to $T_{\mathrm{SR}}$. 


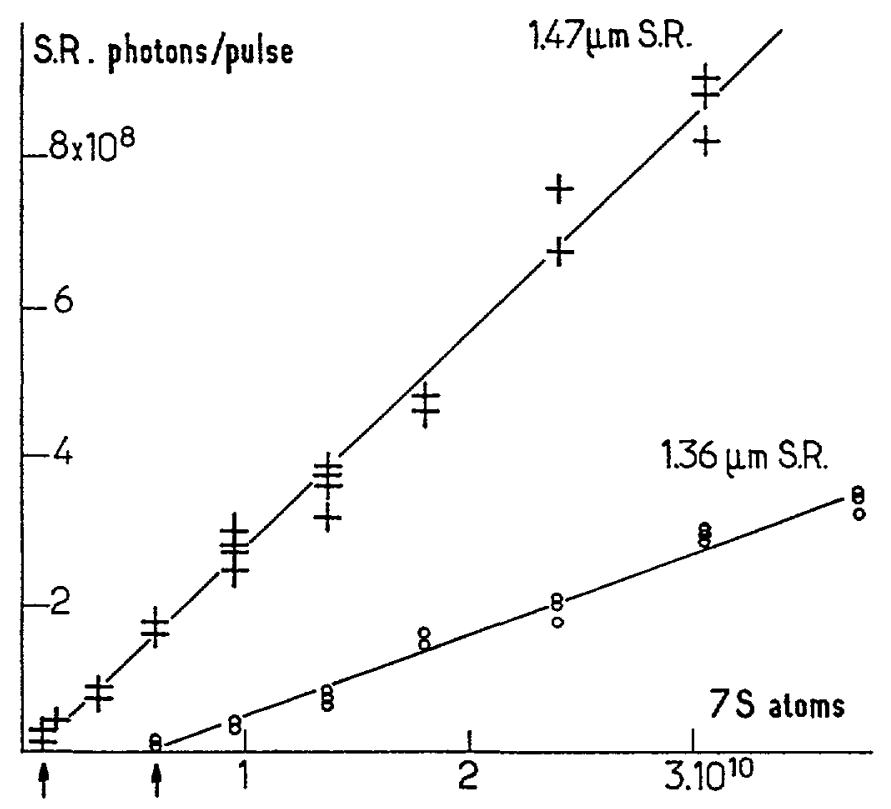

Fig. 11. - Number of emitted photons per pulse in the case of spontaneous superradiance, as a function of the number of $7 \mathrm{~S}$ atoms excited in a transverse $E$-field. Crosses : emission to $6 \mathrm{P}_{3 / 2}$; circles : emission to $6 \mathrm{P}_{1 / 2}$ (selected by a filter). $n_{\mathrm{Cs}}=1.4 \times 10^{14} \mathrm{at} / \mathrm{cm}^{3}$. Exc. pulse $1 \mathrm{~mJ}$, resonant for $F=4 \rightarrow F=4$; beam diameter $1 \mathrm{~mm}$.

4.2 MANIFESTATION OF THE TRIGGERED SUPERRADIANCE. - When triggered by an external resonant field, the superradiant emission is produced earlier and shows reproducibility in the delay, duration, and height of the emitted pulse, which contrasts with the large fluctuations characteristic of spontaneous superradiance. As in the latter case, one can shorten the delay by increasing the gain of the medium. However, one can also vary it by changing either the probe beam intensity or the time at which the probe is switched on if superradiance has not occurred previously.

The recording in figure $13 \mathrm{a}$ represents a superradiance pulse that precedes the opening of the optical switch. Nevertheless, in the total absence of the probe beam the pulse would not have been produced. In fact conditions are such that photons transmitted by the closed optical switch (a few thousandths of the incident flux, or $\leqslant$ one microwatt) are enough to trigger the detected pulse. The latter occurs before the end of the pump pulse and its duration is short ( $\$ 4 \mathrm{~ns}$ ). Unlike in the case of spontaneous superradiance, the triggered pulse almost completely depopulates the $7 \mathrm{~S}$ level : when the switch is opened, only a small gain pulse, of longer duration, is emitted, associated with a rebuildup of $7 \mathrm{~S}$ population after the superradiant pulse.

Triggering of the superradiance has already been studied previously [28]. The conditions realized in the present experiment nonetheless have a certain originality due to:

- the pumping of the excited state by a highly forbidden transition, which permits precise control over a wide range of gain. Varying the electric field acts only on the population inversion without modifying the other important parameters, in particular the phase difference between atomic dipoles;

- the use of the optical switch which in a certain gain range allows us to control the delay of 


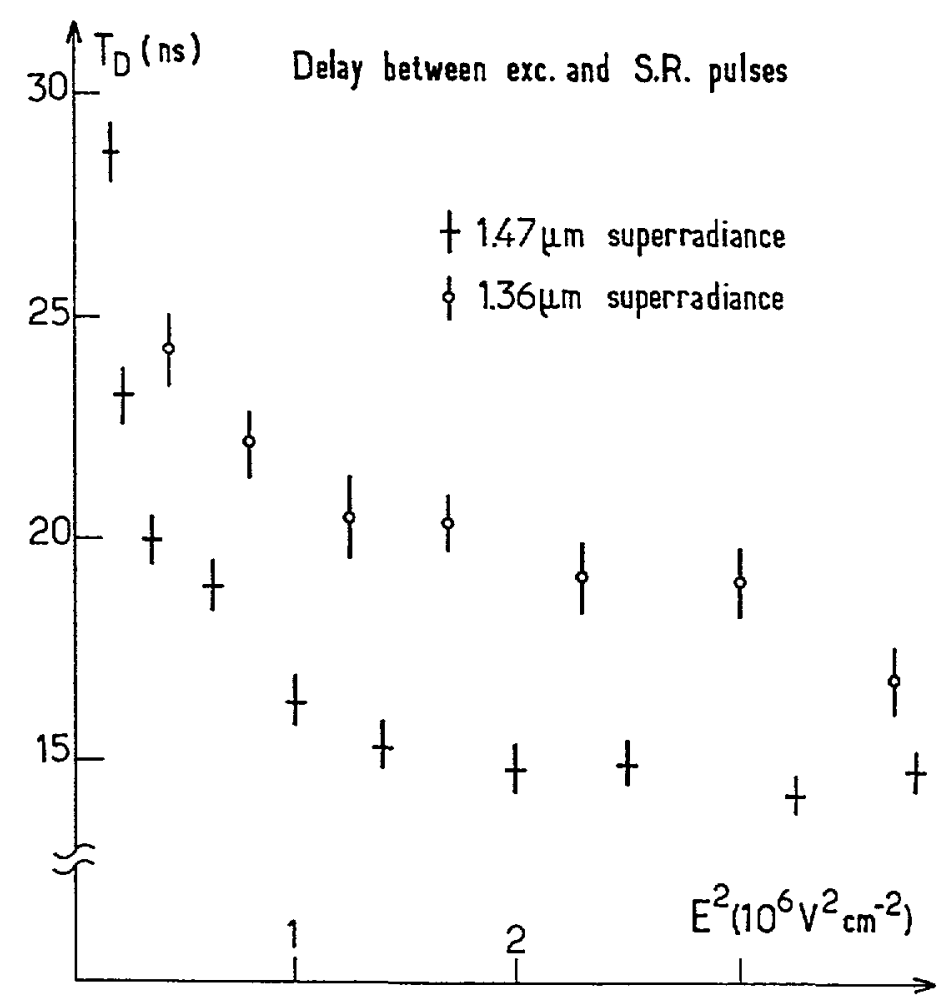

Fig. 12. - Delay of the spontaneous superradiance, as a function of the square of the electric field. Same conditions as in figure 11.
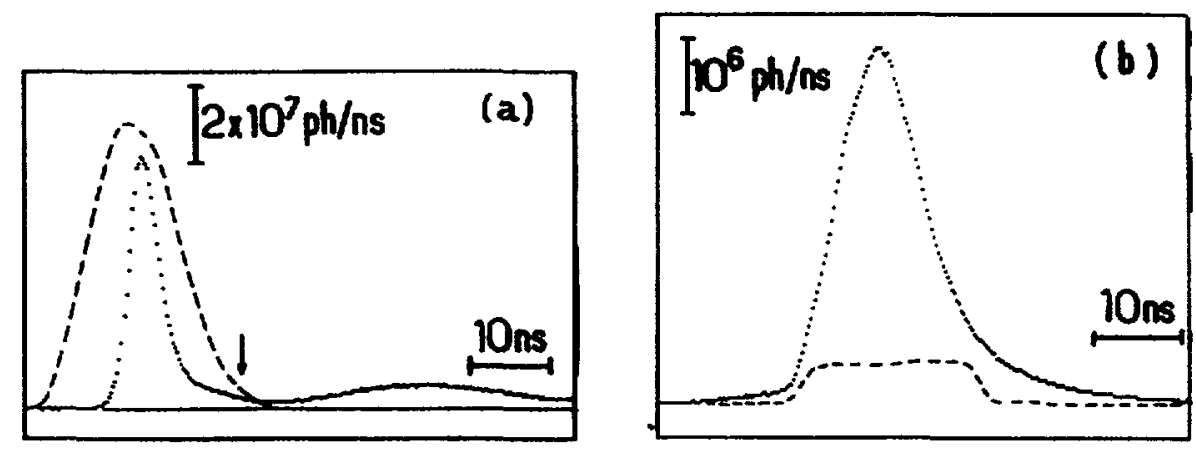

Fig. 13. - Examples of triggered superradiance (SR) pulses : (a) SR pulse triggered by the probe photons leaking from the closed optical switch, followed by a small gain pulse at switch opening (pointed by the arrow). The dashed line represents the pump pulse. Triggering probe power $\sim 0.4 \mu \mathrm{W}$. Transverse $E$-field $1 \mathrm{kV} / \mathrm{cm}$. $n_{\mathrm{Cs}} \simeq 1.8 \times 10^{14} \mathrm{at} / \mathrm{cm}^{3}$. Exc. pulse $0.6 \mathrm{~mJ}$. (b) $\mathrm{SR}$ triggered by the probe gate represented in dash $(80 \mu \mathrm{W}-20 \mathrm{~ns}) . E=400 \mathrm{~V} / \mathrm{cm}$, transverse. 
the emission, as long as the switch is opened before the superradiance has time to spontaneously occur. In this respect, the gain pulse shown in figure $13 \mathrm{~b}$ is a limiting case : the signal actually starts before the switch is opened, this is the beginning of a pulse triggered by the leak of the switch. It has the virtue of preparing the build-up of the dipole so that when the switch is opened, full triggering of the superradiant pulse occurs without delay.

4.3 CONTINUOUS PASSAGE FROM LINEAR AMPLIFICATION TO TRIGGERED SUPERRADIANCE. OPTIMIZATION OF THE PROBE BEAM INTENSITY. - The existence of different amplification regimes has important consequences when optimizing the probe intensity for the physical effect we wish to observe.

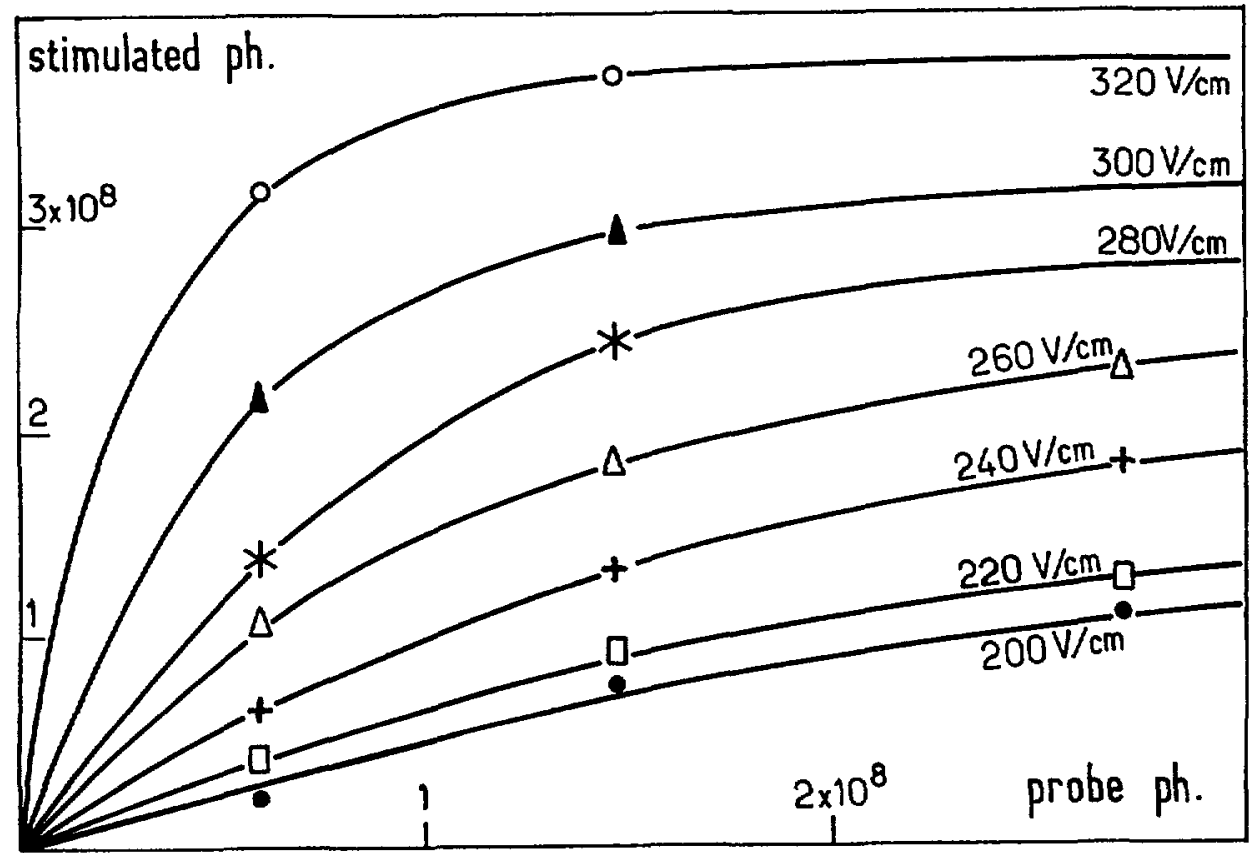

Fig. 14. - Saturation of the amplification signal by the probe beam for several values of the transverse $E$-field : number of induced photons vs. number of incident probe photons (20 ns probe gate). $n_{\mathrm{Cs}} \simeq 7 \times 10^{14} \mathrm{at} / \mathrm{cm}^{3}$. Exc. pulse $0.4 \mathrm{~mJ}$. The asymptots scale as $E^{2}$, i.e. the number of $7 \mathrm{~S}$ excited atoms.

To efficiently depopulate the $7 \mathrm{~S}$ level it would at first sight seem necessary to use a saturating probe (i.e. saturating parameter $\sim 1$ in the steady state). When the density of excited atoms is high enough to cause superradiance, the transition dipole is the collective dipole of the ensemble of atoms. It is then shown [26] that the intensity necessary to completely depopulate the $7 \mathrm{~S}$ state is much lower. The experimental study of the amplification as a function of the probe beam intensity and of the electric field controlling the number of excited atoms demontrates the continuous passage from nearly linear amplification to triggered superradiance, or the passage from individual to collective atomic emission. The former is only likely if the probe beam saturates the atomic transition, the latter requires a much lower flux. This is quite obvious in figure 14 : in a $200 \mathrm{~V} / \mathrm{cm}$ field, one needs $1.5 \times 10^{8}$ probe photons to reach half of the 
maximum signal, while at $320 \mathrm{~V} / \mathrm{cm}$ (i.e. with 2.6 times more excited atoms) $2 \times 10^{7}$ probe photons are sufficient.

\section{Enhancing polarization dependent signals.}

When we first proposed using stimulated emission to detect very small angular anisotropies in the $7 \mathrm{~S}$ state [10], we pointed out an intriguing feature of the method : when the vapor becomes optically thick for the probe, the polarization dependent signal can become more amplified than the intensity of the probe. This opens up the possibility of using the optical medium itself to enhance the effect of a minute anisotropy of its own. In this section our purpose is to present illustrations of the effect.

5.1 ENHANCEMENT OF A CIRCULAR DICHROISM SIGNAL. - When the 7S state possesses an orientation it emits with a larger probability photons of a given sign of circular polarization. As a result, a linearly polarized incoming probe acquires a circular polarization as shown in section 2. If the vapor is optically thick, further interaction of the altered probe beam with the vapor gives an additional reason to favour the same cicular polarization state at each subsequent induced emission process. An autoamplification of the probe circular polarization during propagation is thus expected at high gain. This effect has been tested for a 7S orientation of $8 \times 10^{-2}$ created in the $4 \rightarrow 4$ transition where large gains are easily obtainable in a transverse E-field. In the case of the $F=4 \rightarrow F^{\prime}=5$ probe transition, the measured amplification anisotropy $\left(S_{x}-S_{y}\right) /\left(S_{x}+S_{y}\right)$ increases clearly as soon as the amplification factor exceeds unity. In extreme situations of triggered superradiance (transverse field $E=560 \mathrm{~V} / \mathrm{cm}$, interaction length $=3 \mathrm{~cm}$ ) this anisotropy even reaches 0.3 , while it is expected to be $5 \times 10^{-2}$ in the linear amplification regime, a six-fold enhancement.

5.2 ENHANCEMENT OF A LINEAR DICHROISM SIGNAL. - Such an effect would be of particular interest for increasing the sensitivity to a parity violation effect [10]. To demonstrate the enhancement possibility we have chosen the most intense probe transition $\left(7 \mathrm{~S}_{1 / 2} F=\right.$ $4 \rightarrow 6 \mathrm{P}_{3 / 2} F^{\prime}=5$ ), and the probe linear polarization which gives rise to the smallest gain $\left(\epsilon_{\mathrm{ex}} / / \epsilon_{\mathrm{pr}}\right)$. The signal monitored as a function of gain was the $\theta_{\mathrm{F}}$-odd polarization signal associated with a tilt $\theta_{\mathrm{F}}$ of $\epsilon_{\mathrm{ex}}$ (see Fig.7 in Sect. 2). While the probe propagates through the optically thick and anisotropic vapor, its polarization is driven towards the orthogonal direction of larger gain. For this reason we expect an enhancement of the $\theta_{\mathrm{F}}$-odd signal at increasing gains. We have made the measurements in a regime of high gain ( $E$-field controlled) and very weak probe saturation. The detected amplification anisotropy plotted in figure 15 does show an increase. At the largest amplifications explored, it exceeds the value anticipated in the linear regime by a factor of 4 .

Such enhancements caused by non linear amplification, though very attractive, raise an important question : are they compatible with absolute and precise measurements ? We have brought a positive answer to this question [13] : the trick is to measure the ratio between two signals subjected to the same enhancements, one of them being the $\theta_{\mathrm{F}}$-odd dichroism signal obtained with a well-calibrated $\theta_{F}$ angle.

Let us underline here how important the choice of the detection transition is for making it possible to reach the non linear regime. If, instead of the $6 \mathrm{~S}-7 \mathrm{~S}-6 \mathrm{P}$ system, we would have chosen a $6 \mathrm{~S}-7 \mathrm{~S}-n \mathrm{P}$ system with $n \geq 10$ characterized by a much more convenient probe wavelength, the smallness of the 7S-nP oscillator strength would have confined the investigated 


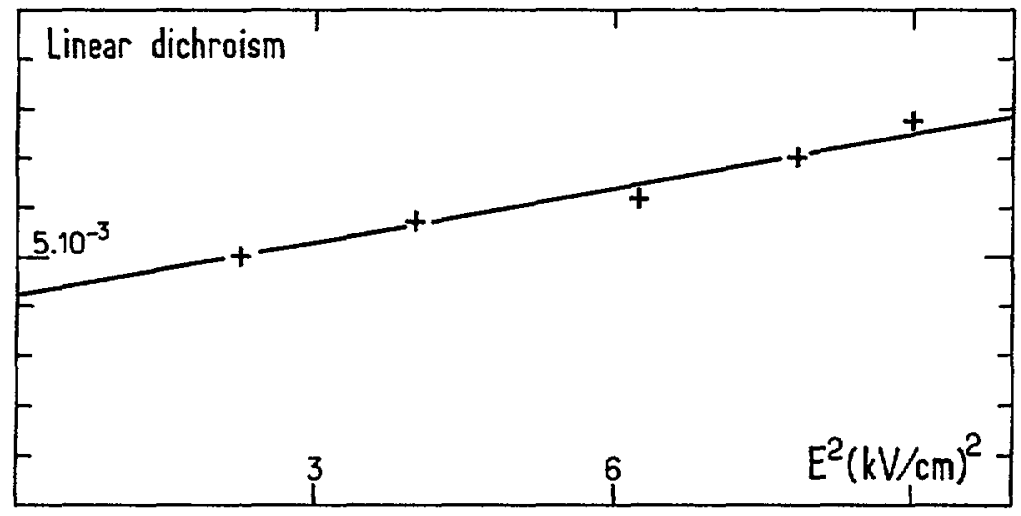

Fig. 15. - Enhancement of the amplification anisotropy due to linear dichroism at high gain and very low input probe saturation. Probe laser tuned to $4 \rightarrow 5$. $\theta_{F}=6.2 \times 10^{-3}$ rad. Other conditions as in figure 7.

domain well below the non linear regime. Then, instead of enhancing polarization dependent signals we would have met the serious problem of signal dilution.

\section{Relative position of the beams.}

In pump-probe experiments good superposition of the beams is obviously of prime importance for a good signal. It is even more important when, as in our case, one deals with polarization measurements of a small anisotropy of the vapor. Then in addition, the beams must preserve the axial, or even rotation symmetry : each beam must be symmetric and their superposition must be symmetric as well. For example our experiment is in principle symmetric under rotation around the common axis of the electric field and beams. This feature may be used to track possible imperfections such as transverse $\mathbf{E}$ or $\mathbf{H}$ fields, but this requires that the cylindrical beams be coaxial. The beams are aligned by nearly exact superposition of their centers at two points located before and after passage through the cell using four quadrant detectors, in a way inspired from [29].

On the contrary, breaking the symmetry on purpose can be useful to test some phenomena. It is known that an intense pulse of light propagating in an atomic vapor at high densities can generate a radially symmetric electric field [30]. In the case of our E-field controlled transition this field can lead to spurious effects, so its study is of special importance. With the idea of probing a radial field we have broken the symmetry of the interaction region in one of two ways : i) displacing the pump beam from the probe beam by a known space translation of the order of a beam radius, ii) masking half of the probe beam at the output of the cell so as to observe only one half of the interaction region. Then any effect proportional to a radial electric field no longer cancels out but instead shows up in full magnitude. This has allowed us to measure the radial field induced by the light pulse in certain conditions. This experimental study will be reported elsewhere. 


\section{Conclusion.}

This paper illustrates the flexibility and advantages of the "active detection" of excited atoms. A probe beam triggers stimulated emission from the excited state. The detection efficiency is of the order of unity, unlike the case of fluorescence detection where the angular acceptance for emitted photons is always limited, particularly if fluorescence polarization analysis is necessary. Here, active detection is demonstrated for atoms which have been excited by a pump pulse resonant for the highly forbidden $6 \mathrm{~S} \rightarrow 7 \mathrm{~S}$ transition of cesium. The very weak oscillator strength can be enhanced in the range $10^{-14}-10^{-11}$ by applying a static electric field. In spite of the very small optical density at the pump wavelength, the use of intense pump pulses leads to large optical density for the probe ( 1 or even more). By adjusting the electric field, and hence the upper state population, one can observe at will small or large probe amplification, and approach gradually the conditions of superradiant emission.

Our bidifferential polarimetry technique performs a double task : it eliminates the pulse to pulse fluctuations inherent to our excitation mechanism, and it provides an unambiguous discrimination of the birefringence (or dichroism) effects associated with the excited atoms, against other possible sources. With respect to previous pulsed experiments, as those which have demonstrated superradiance in the optical domain, the progress achieved for this forbidden transition provides a signal quality now quite comparable to what is for instance currently obtained in polarization spectroscopy in allowed transitions with $\mathrm{cw}$ lasers. Futhermore for large probe amplifications, we have demonstrated enhancement of the polarization modifications experienced by the probe beam with respect to a linear regime.

An additional advantage of this active detection scheme, which was designed in the perspective of precise measurements of atomic parity violation, is that it may benefit, in the longer term, from further sensitivity improvement associated with a squeezed probe beam [31], or twin beams [32].

\section{References}

[1] Kastler A., J. Phys. France 11 (1950) 255.

[2] Brossel J., Kastler A., Winter J.M., J. Phys. France 13 (1952) 668;

KASTLER A., Science 158 (1967) 214.

[3] Dehmelt H.G., Phys. Rev. 105 (1957) 1487.

[4] Brossel J., Optique et Electronique Quantique, Les Houches (Gordon and Breach, N.Y.), (1964) p.187. As a spectacular example of Dehmelt pumping, see WAGSHUL M.E., and CHUPP T.E., Phys. Rev. A 40 (1989) 4447.

[5] Dehmelt H.G., Phys. Rev. 105 (1957) 1924.

BeLl W.E. and Bloom A.L., Phys. Rev. 107 (1957) 1559.

[6] Gozzini A., C.R. Acad. Sci. 255 (1962) 1905.

[7] Happer W., Rev. Mod. Phys. 44 (1972) 169.

[8] Bouchiat M.A., Guena J. and Pottier L., J. Phys. France 46 (1985) 1897;

Drell P. and Commins E.D., Phys. Rev. A 32 (1985) 2196;

Noecker M., Masterson B. and Wieman C., Phys. Rev. Lett. 61 (1988) 310;

WOLFENDEN T., BAIRD P. and SANDARS P., Europhys. Lett. 15 (1991) 731;

Macpherson M., Zetie K., Warrington R., Stacey D. and Hoare J., Phys. Rev. Lett. 67 (1991) 2784. 
[9] Bouchiat M.A., Guena J., Hunter L. and Pottier L., Phys. Lett. B117 (1982) 358; Phys. Lett. B134 (1984) 463; J. Phys. France 47 (1986) 1709.

[10] Bouchiat M.A., Jacquier Ph., Lintz M. and Pottier L., Opt. Commun. 56 (1985) 100.

[11] Guena J., Lintz M., Jacquier Ph., Pottier L. and Bouchiat M.A., Opt. Commun. 62 (1987) 97.

[12] Bouchiat M.A., Guena J., Jacquier Ph., Lintz M. and PotTier L., J. Phys. France 50 (1989) 157.

[13] Bouchiat M.A., Guena J., Jacquier Ph., Lintz M. and Pottier L., Opt. Commun. 77 (1990) 374.

[14] Xie W., Ritter A., Harkin C., Kasturi K., Dai H.L., J. Chem. Phys. 89 (1988) 7033;

Frye D., Liou H.T. and DaI H.L., Chem. Phys. Lett. 133 (1987) 249;

VacCaro P.H., Kinsey J.L., Field R.W. and DaI H.L., J. Chem. Phys. 78 (1983) 3659;

J. Opt. Soc. Am. 77 (1990) 1805.

[15] Ducas T.W., Littman M.G., Zimmerman M.L., Phys. Rev. Lett. 35 (1975) 1752;

LANGe W. and Mlynek J., Phys. Rev. Lett. 40 (1978) 1373.

[16] Bouchiat M.A. and Bouchiat C., J. Phys. France 36 (1975) 493;

Bouchiat M.A. and Pottier, L., J. Phys. Lett. France 36 (1975) L-189.

[17] Hoffnagle J. et al., Phys. Lett. A85 (1981) 143;

BovChiAT M.A. et al., Opt. Commun. 45 (1983) 35;

Gilbert S.L. et al., Phys. Rev. A27 (1983) 581.

[18] Herrmann P., Hoffnagle J., Schlumpf N., Telegdi V. and Weis A., J. Phys. B19 (1986) 1607.

[19] Wieman C. and Hansch T.W., Phys. Rev. Lett. 36 (1976) 1170.

[20] Guena J. et al., Opt. Commun. 71 (1989) 6.

[21] ShurclifF W.A., Polarized Light (Harvard University Press, Cambridge 1962) p.118 et Appendice 2.

[22] Heavens O.S., J. Opt. Soc. Am. 51 (1961) 1058.

[23] Collins C.B. et al., J. Chem. Phys. 74 (1981) 1053 and 1067.

[24] Maggillivray J.C. and Feld M.S., Phys. Rev. A14 (1976) 1169.

[25] Pillet P., Thèse de 3ème cycle, Université Paris VI (1977), unpublished;

Gross M., Thèse d'Etat, Université Paris VI (1980) unpublished.

[26] Gross M. and HARoche S., Phys. Rep. 93 (1982) 302.

[27] Pavolini D., Crubellier A., Pillet P., Cabaret L. and Liberman S., Phys. Rev. Lett. $54(1985) 1917$.

[28] Carlson W.W., Jackson D.J., Schawlow A.L., Gross M. and Haroche S., Opt. Commun. $32(1980) 350$.

[29] Grafstrom S., Harbarth U., Kowalski J., NeumanN R. and Noehte S., Opt. Commun. 65 (1988) 121.

[30] MiYazaki K., Sato T. and Kashiwagi H., Phys. Rev. Lett. 43 (1979) 1154;

Bethune D.S., Phys. Rev. A23 (1981) 3139.

[31] Grangier P., Slusher R., Yurke B. and Laporta A., Phys. Rev. Lett. 59 (1987) 2153.

[32] Heidmann A. et al., Phys. Rev. Lett. 59 (1987) 2555;

Aytur O. and Kumar P., Phys. Rev. Lett. 65 (1990) 1551. 